\title{
Questes
}

\section{Semer le doute : le problème cosmogonique chez Maïmonide}

\section{Diana Di Segni}

\section{(2) OpenEdition}

1 Journals

\section{Édition électronique}

URL: http://journals.openedition.org/questes/942

DOI : $10.4000 /$ questes. 942

ISSN : 2109-9472

\section{Éditeur}

Les Amis de Questes

\section{Édition imprimée}

Date de publication : 15 mars 2012

Pagination : 33-47

ISSN : 2102-7188

\section{Référence électronique}

Diana Di Segni, « Semer le doute : le problème cosmogonique chez Maïmonide », Questes [En ligne], 23 | 2012, mis en ligne le 01 janvier 2014, consulté le 19 avril 2019. URL : http:// journals.openedition.org/questes/942; DOI : 10.4000/questes.942 


\section{Semer le doute : \\ le problème cosmogonique chez Maïmonide}

\section{Diana DI SEGNI}

Le doute - ainsi que son étymologie le montre - concerne celui qui, se trouvant face à deux possibilités, ne sait laquelle choisir, et c'est justement à ce genre de lecteur qu'est adressé le Guide des Égarés ${ }^{1}$. Dans l'introduction à son ouvrage, Maïmonide affirme vouloir éclaircir les doutes de son disciple qui se heurte à la contradiction entre philosophie et religion, incapable de substituer à la simple foi la conviction rationnelle. Il faut remarquer que la nuance sémantique donnée par la traduction française $\mathrm{du}$ titre de l'ouvrage ne rend pas explicitement les implications de la dichotomie : alors que les «égarés » ont perdu leur chemin, les Hāyryn et les Nevoukhim du titre original - dans sa version en arabe et en hébreu ${ }^{2}-$ sont strictement liés par leur racine au sens du doute, que préserve le titre latin dans ses différentes variantes à l'intérieur de la tradition manuscrite, comme le montre notamment le titre Dux neutrorum vel dubiorum vel perplexorum ${ }^{3}$. Toutefois, malgré la déclaration d'intention de l'auteur, le raisonnement maïmonidéen ne révèle pas de façon directe ses véritables positions. Le doute transparaît aussi dans le développement de

\footnotetext{
${ }^{1}$ Moïse MaÏmonide, Le Guide des égarés : traité de théologie et de philosophie, Salomon MunK (trad.), Paris, G.-P. Maisonneuve \& Larose, 1970 (1 ${ }^{\text {ère }}$ éd. 1856-1870).

${ }^{2}$ Rabbenu Moshé Ben MAIMun, Dalālat al-Hāyryn, Salomon MunK et Issachar Joel (éd.), Jérusalem, J. Junovitch, 5691 (=1931); Rabbenu Moshé BEN MAIMUN, Moré Nevukhim, Yehuda IBN SHMUEL (éd.), Jérusalem, Mossad Harav Kook, 2000. Selon Amnon GILEADI, «A Short Note on the Possible Origin of the Title 'Moreh ha-Nevukhim' », Le Muséon, 97 (1984), p. 159-161, le titre arabe aurait été inspiré par un ouvrage de al-Ghazali, Revivification des sciences de la foi, dans lequel Dieu est appelé «guide des perplexes » (en arabe dalīl al-mutahā'irīn). La racine hébraïque est liée à l'idée de désarroi, de confusion (Isaïe, XXII, 5) et, en hébreu moderne, le mot signifie « embarras ».

${ }^{3}$ Il n'existe pas d'édition critique du texte latin. Cela fait précisément l'objet de la thèse de doctorat que je prépare sous la direction d'Andreas Speer et Loris Sturlese à l’Université de Cologne, en cotutelle avec l’Université du Salento (Lecce).
} 
l'argumentation, parce que Maïmonide se propose de déguiser la vérité aux yeux des lecteurs non-initiés. Dans cette brève présentation, nous nous intéresserons au problème cosmogonique tel qu'il est exposé dans le Guide ; en vue de cela, il faudra toujours garder à l'esprit cette double perspective du doute - celle des destinataires ainsi que celle de l'auteur «mystificateur ».

Après une brève esquisse de la méthode maïmonidéenne, on analysera dans ce cadre le problème de la création du monde, puis l'on recherchera une solution possible à cette question théorique dans le rapport du système cosmogonique à la prophétologie. Il sera indispensable de se référer constamment au texte - dans la tentative, peut-être vaine, d'en déceler le vrai sens - en raison de l'obscurité choisie par un Maïmonide soucieux de cacher ses véritables opinions, et de la complexité de la question de la création du monde, qui tient principalement à la difficulté du mode argumentatif.

\section{La méthode maïmonidéenne}

Clarifier les doutes liés aux contradictions entre philosophie et religion est le but du Guide, qui se propose d'aider le disciple - dont les qualités sont décrites par Maïmonide dans l'épître dédicatoire - qui doit être «parfait dans sa religion et dans ses mœurs, qui a étudié les sciences des philosophes ${ }^{4}$ et qui doit avoir « une grande passion pour l'étude » et « un amour très prononcé pour les choses spéculatives $»^{5}$. L'étude des sciences astronomiques, mathématiques et logiques est un réquisit nécessaire avant de pouvoir être initié à la connaissance des secrets des livres prophétiques, car ce sont ceux qui les étudient, surtout, qui doivent

\footnotetext{
${ }^{4}$ Moïse MAÏMONIDE, Le Guide des égarés, éd. cit., introduction, p. 7.

${ }^{5}$ Ibid., introduction, p. 4.
} 
affronter des contradictions perçues entre la loi biblique et la science naturelle.

Maïmonide débute ainsi son argumentation par l'éclaircissement des termes bibliques équivoques; le langage vétérotestamentaire, riche en métaphores, pourrait engendrer, pris dans son sens littéral, un état de «perplexité » ${ }^{6}$. Cependant, la contradiction n'est qu'apparente, philosophie et religion n'étant pas supérieures l'une à l'autre. Ce n'est qu'après avoir éclairci la vraie signification du langage biblique que l'on comprend qu'il s'agit simplement de deux manières d'exprimer la même vérité.

Le plan du Guide n'est toutefois pas aussi clair que son propos : de nombreux interprètes de la pensée maïmonidéenne ont proposé plusieurs reconstructions, dont la plus célèbre a été formulée par Leo Strauss dans son essai introductif à la traduction anglaise du Guide ${ }^{7}$. Les secrets de la loi que Maïmonide veut déceler ne sont pas faits pour être expliqués en public et ne peuvent être dévoilés qu'aux hommes initiés. C'est pourquoi l'auteur distingue ses arguments au lieu de les éclaircir et les disperse tout au long de son ouvrage, les chapitres n'étant pas rangés par ordre ni d'une manière suivie :

Ces éléments mêmes ne se trouvent pas [...] rangés par ordre $[\ldots]$ mais au contraire, disséminés et mêlés à d'autres sujets qu'on voulait expliquer; car mon but est (de faire en sorte) que les vérités y soient entrevues, et qu'ensuite elles se dérobent, afin de ne pas être en opposition avec le but divin, auquel d'ailleurs il serait impossible de s'opposer, et qui a fait que les vérités qui ont particulièrement pour objet

\footnotetext{
${ }^{6}$ Ibid., introduction, p. 7-8.

7 Voir l'introduction de Leo STRAUSS, «How to begin to study The Guide of Perplexed » à Moses MAIMONIDES, The Guide of Perplexed, Shlomo PINES (éd.), Chicago, The University of Chicago Press, 1963, vol. I, p. XI-LVI. Il faut remarquer que le Guide est divisé en trois livres, et contient cent soixante-dix-huit chapitres. Strauss organise le contenu de l'ouvrage par thèmes, en structurant les trois livres en deux parties, sept sections et trente-huit sous-sections.
} 
de faire comprendre Dieu fussent dérobées au commun des hommes $[\ldots]^{8}$

Pour comprendre ses positions, il faut résoudre le «puzzle ${ }^{9}$ en repérant des connexions entre les chapitres, ainsi que le suggère notre auteur $^{10}$. Certaines étapes de l'argumentation ont même été intentionnellement cachées, afin de dissimuler au peuple les secrets et les contradictions que présente l'ouvrage. Si l'homme savant n'est pas troublé par ces contradictions apparentes, l'homme simple d'esprit ne saurait les comprendre, aussi ce dernier ne doit-il pas les apercevoir :

Car quelquefois on se voit forcé, en émettant une opinion, de s'exprimer de manière à affirmer une certaine proposition, tandis que dans un autre endroit on se voit forcé de s'exprimer de manière à affirmer une proposition qui se trouve en contradiction avec la première. Le vulgaire ne doit d'aucune manière s'apercevoir de l'endroit où existe la contradiction, et l'auteur quelquefois cherche toute sorte d'expédients pour la dérober. ${ }^{11}$

\section{Le problème de la création du monde}

Le même procédé est employé dans le cas de la question de la création du monde. Maïmonide, en effet, doit sur ce point faire face à deux paradigmes différents : tandis que les philosophes aristotéliciens adoptent la théorie de l'éternité du monde, notre auteur ne peut pas renoncer à l'idée biblique d'une libre volonté divine dans l'acte de la création. Dans le

\footnotetext{
${ }^{8}$ Moïse MAÏMONIDE, Le Guide des égarés, éd. cit., introduction, p. 9-10.

${ }^{9}$ La question cosmogonique peut s'apparenter à un «puzzle » à reconstruire, selon le terme d'un célèbre article qui a traité le problème du rapport entre cosmogonie et prophétie chez Maïmonide. Cf. Warren Zev HARVEY, «A Third Approach to Maimonides Cosmogony-Prophetology Puzzle », Harvard Theological Review, 74, 3 (1981), p. 287-301. Voir aussi Roberto GATTI, «Il problema filosofico della creazione nella Guida dei perplessi di Maimonide», Materia Giudaica, IX, 1-2 (2004), p. 175-181.

${ }_{10}^{10}$ Moïse MAÏMONIDE, Le Guide des égarés, éd. cit., introduction, p. 23.

${ }^{11}$ Ibid., p. 29.
} 
Guide, il expose trois opinions distinctes concernant la création, sans préciser explicitement quelle est la sienne ${ }^{12}$.

Selon la première opinion - admise par « tous ceux qui croient dans la loi de Moïse $»^{13}$ - le monde a été créé à partir d'un absolu non-existant par la libre volonté de Dieu, qui ne s'est aidé d'aucun autre élément, le temps n'existant pas non plus avant la création :

Que l'univers, dans sa totalité [...] c'est Dieu qui l'a produit du néant pur et absolu ; qu'il n'avait existé (d'abord) que Dieu seul et rien en dehors de lui, ni ange, ni sphère, ni ce qui est à l'intérieur de la sphère céleste; qu'ensuite il a produit tous ces êtres, tels qu'ils sont, par sa libre volonté et non pas de quelque chose ; enfin, que le temps lui-même aussi fait partie des choses créées. ${ }^{14}$

L'opinion selon laquelle la création succède à un absolu non-existant ne coïncide pas exactement avec l'idée biblique de la création à partir du néant, puisque le nihil pourrait avoir le statut ontologique d'un étant et que l'acte créateur correspondrait alors à un acte d'information éternelle de la matière ${ }^{15}$. Cette première opinion exclut donc complètement la théorie de la création éternelle alors que la création ex nihilo pourrait l'admettre - nous y reviendrons.

Suivant un mode argumentatif aristotélicien, Maïmonide présente plusieurs difficultés s'opposant à cette première opinion : certains éléments - tels que le mouvement, le temps, la matière première et la matière céleste - ne peuvent pas être créés ${ }^{16}$. Le premier mouvement ainsi que la

\footnotetext{
${ }^{12}$ Ibid., II, 13.

${ }^{13}$ Ibid. II, 13, p. 104.

${ }^{14}$ Ibid., II, 13, p. 104-105.

${ }^{15}$ L'expression hébraïque tohu vavohu (Genèse, I, 1) - traduite par « informe et vide »est assez ambiguë. Elle pourrait signifier la matière, créée, mais préexistante à la création du monde. Cf. Talmud Bavlì, Hagigah 12a.

${ }^{16}$ Pour le mouvement, voir ArISTOTE, La Physique, Annick STEVEns (trad.), Paris, Vrin, «Bibliothèque des textes philosophiques », 1999, VIII, 1, 251a 3-5, p. 272 et Moïse MAÏMONIDE, Le Guide des égarés, éd. cit., II, 14, p. 115. Pour la matière première, voir ARISTOTE, La Physique, éd. cit., I, 3, 192a 28-30, p. 95 et Moïse MAÏMONIDE, Le Guide des égarés, éd. cit., II, 14, p. 115. Pour la matière céleste,voir
} 
matière première doivent nécessairement être éternels car, si tel n’était pas le cas, la série des mouvements et des matières remonterait à l'infini. De la même manière le temps, qui est attaché au mouvement, et la matière céleste, qui n'a pas de contraire, ne sont pas sujets à la génération et à la corruption. De plus, une production quelconque requiert un substrat antérieur de possibilité : au cas où la création du monde serait possible, un autre élément sur lequel fonder cette même possibilité d'existence serait nécessaire. Par conséquent, une création à partir d'un absolu non-existant est dans ce cas inconcevable, tandis que, si la création était nécessaire, l'action divine aurait été contrainte par cette nécessité. Enfin - ce qui est presque tautologique - dans le cas d'une impossibilité de la création, il n'y aurait tout simplement pas de monde $^{17}$. Un raisonnement analogue concerne Dieu qui - s'Il a créé le monde dans le temps - a dû être un agent in potentia et ensuite un agent in actu, le passage de la puissance à l'acte impliquant l'existence d'un autre élément qui a dû interférer avec son $\operatorname{action}^{18}$.

Notre auteur introduit ensuite la notion d'infini par accident, à savoir un infini résultant d'une succession d'accidents, comme dans le cas de la génération : tandis qu'un être humain naît, un autre meurt, et la série qui relie un père à son fils, son petit-fils, etc. peut être qualifiée d'infinie ${ }^{19}$.

ARISTOTE, Traité du ciel, suivi du Traité pseudo-aristotélicien du monde, Jacques Tricot (trad.), Paris, Vrin, «Bibliothèque des textes philosophiques », 1990 ( 1 ère éd. 1948), I, 3 270a 16-19, p. 10 et Moïse MAÏMONIDE, Le Guide des égarés, éd. cit., II, 14, p. 116.

${ }^{17}$ Moïse MAÏMOnide, Le Guide des égarés, éd. cit., II, 14, p. 117-118. Cf. ARISTOTE, La Physique, éd. cit., VIII, 1, 251a 10-16, p. 269.

${ }_{18}^{18}$ Moïse MaÏMONIDE, Le Guide des égarés, éd. cit., II, 14, p. 119.

19 «Ceux qui prétendent avoir démontré l'éternité du monde soutiennent que le temps est infini, et il ne s'ensuit pour eux rien d'inadmissible, car, à mesure qu'une partie du temps se produit, une autre qui la précédait a disparu ; et il en est de même, selon eux, de la succession des accidents qui surviennent à la matière jusqu'à l'infini, sans qu'il s'ensuive rien d'inadmissible, puisqu'ils n'existent pas tous simultanément, mais successivement, ce dont l'impossibilité n'a point été démontrée » (ibid., I, 73, p. 414-415). 
Cependant, «tous les philosophes dont nous avons entendu parler, ou dont nous avons vu les paroles $»^{20}$ admettent la seconde opinion sur la création, à savoir la cosmogonie platonicienne, selon laquelle un étant ne peut pas se produire à partir du non-existant et ensuite se réduire au néant. L'univers a donc été créé, mais à partir d'une matière éternelle :

Il est inadmissible, disent-ils, que Dieu produise quelque chose du néant, et il n'est pas non plus possible, selon eux, qu'une chose soit réduite au néant (absolu). [...] Attribuer à Dieu la faculté de (faire) pareille chose, ce serait, selon eux, comme si on lui attribuait la faculté de réunir au même instant les deux contraires. [...] Ils croient donc qu'il existe une matière qui est éternelle comme Dieu; que lui, il n'existe pas sans elle, ni elle sans lui. ${ }^{21}$

La loi n'est pas complètement détruite en ses fondements en vertu de ce système, car il implique l'intervention d'un artifex, qui organise la matière éternelle et informée tout en se distinguant du créateur, lequel agit par sa propre et libre volonté sans être contraint par une matière préexistante. Certains textes rabbiniques rapportant l'idée d'une matière éternelle ou de l'existence d'un étant coéternel avec Dieu pourraient être mis en relation avec la cosmogonie ici décrite. En effet, on trouve dans le texte maïmonidéen une référence directe au Pirqué de Rabbi Eliezer (Chapitres de Rabbi Éliézer) ${ }^{22}$, un ouvrage composé au VIII ${ }^{\mathrm{e}}$ siècle, qui contient des midrachim ${ }^{23}$ renvoyant pour la plupart au livre de la Genèse ${ }^{24}$. Au troisième chapitre du Pirqué, Éliézer aborde la question de l'origine de

${ }^{20}$ Ibid. II, 13, p. 107.

${ }^{21}$ Ibid., II, 13, p. 107-108.

22 Ibid., II, 26, p. 200. Éliézer BEn HyRCANOS, Chapitres de Rabbi Eliezer, Éric SMILÉvitch (éd.) et Marc-Alain OUAKNIN (trad.), Verdier, Lagrasse, «Les Dix paroles », 1992.

${ }^{23}$ Le midrach est un procédé rabbinique consistant à compléter les lacunes de la partie narrative de la Bible.

${ }^{24}$ Le Pirqué analyse longuement le Maasé Merkava, le «récit du chariot» (en se référant à Ézéchiel, I). Dans le livre d'Ézéchiel, on trouve la description du trône-chariot de Dieu, conduit par quatre anges. Le Maasé Merkava est considéré par les mouvements mystiques comme l'expression métaphorique des secrets de la création ; c'est pour cela que ce genre d'études était souvent interdit. 
la terre et du ciel, dans un passage considéré comme «étrange » par Maïmonide $^{25}$, car il y est écrit que les cieux viendraient du manteau de Dieu, alors que la terre tiendrait son origine de la neige située au-dessous de son trône. Nous pouvons ici comparer le texte tel qu'il est cité par Maïmonide (à gauche) à l'original même (à droite) :

D'où furent créés les cieux ? Il [Dieu] prit de la lumière de son vêtement et l'étendit comme un drap ; et de là les cieux allèrent se déployant, ainsi qu'il a été dit : «Il s'enveloppe de lumière comme d'un vêtement, il étend les cieux comme un tapis » (Psaumes, CIV, 2). D'où fut créée la terre ? Il prit de la neige de dessous le trône de sa gloire et la lança, ainsi qu'il a été dit : «Car à la neige il dit: "sois terre" »(Job, XXXVII, 6). ${ }^{26}$

\begin{abstract}
D'où furent créés les cieux ? De la lumière de l'habit dont il était vêtu ; il l'ôta, l'étendit comme un manteau, et les cieux se déployèrent continûment jusqu'à ce qu'Il leur dise : «Cela suffit !» C'est ainsi qu'il fut appelé Chaddaï, pour avoir dit «il suffit » à l'adresse des cieux, lesquels aussitôt s'arrêtèrent. Comment savons-nous qu'ils furent créés à partir de la lumière de son vêtement ? Parce qu'il est dit: « Tu T'enveloppes de lumière comme d'un manteau, Tu déplaces les cieux comme une tenture » (Psaumes, CIV, 2).

D'où fut créée la terre ? De la neige qui était sous le Trône de gloire. Il la ramassa et en aspergea les eaux qui se congelèrent aussitôt, et ainsi fut réalisée la poussière de la terre, comme il est dit: "Il dit à la neige : "sois terre" » (Job, XXXVII, 6). ${ }^{27}$
\end{abstract}

On peut remarquer que Maïmonide ne cite pas le passage en entier, et que ses silences sont assez significatifs. Tout d'abord, l'expression «dont il était vêtu » est omise, afin d'éliminer toute formule qui pourrait engendrer une vision anthropomorphique de la divinité. Il manque, deuxièmement, l'idée du déploiement continu des cieux, qui figure aussi dans le traité

\footnotetext{
${ }^{25}$ Moïse MAÏMONIDE, Le Guide des égarés, éd. cit., II, 26, p. 200.

${ }^{26}$ Ibid.

${ }^{27}$ Éliézer Ben Hyrcanos, Chapitres de Rabbi Éliézer, éd. cit., p. 32.
} 
Hagigah du Talmud ${ }^{28}$, selon lequel l'action divine a été nécessaire pour arrêter l'univers qui, après la création, s'étendait perpétuellement. Cette idée d'un univers en expansion, et qui possède sa propre autonomie de sorte qu'il peut grandir infiniment, est absolument réfutée par Maïmonide.

En ce qui concerne la cosmologie, Maïmonide conteste également une autre théorie du Talmud, à savoir que la terre serait environnée au-dessous et au-dessus par les eaux, ce qu'implique le discours de Rabbi Éliézer puisque, en affirmant que Dieu a jeté la neige sur les eaux, il se réfère aux eaux qui se trouvaient au-dessous et qui ensuite se sont congelées pour former la terre.

D'autres sources rabbiniques rapportent l'existence d'un certain nombre d'éléments avant la création : «Sept choses ont été créées avant le monde : la Torah, le repentir, le Jardin d'Eden, la Géhenne, le Trône de la gloire, le Temple, et le nom du Messie », lit-on dans le traité Nedarim du Talmud $^{29}$. Toutefois, Maïmonide semble ne retenir de Rabbi Éliézer que l'idée de la distinction entre la matière des cieux et de la terre, sans paraître remarquer que, selon ce midrach, certains éléments étaient déjà créés. L'impossibilité de la corruption de l'univers est ensuite prouvée par l'analogie avec le Trône divin qui, même s'il a été créé, ne périt pas ${ }^{30}$; il s'agit ici d'une manière de se rapprocher de l'hypothèse de la création éternelle, comme nous le verrons par la suite.

Enfin, la théorie aristotélicienne constitue la troisième opinion présentée par Maïmonide, selon laquelle l'univers vient de Dieu par nécessité, de même que l'intellect produit les intelligibles par nécessité. Par

\footnotetext{
${ }^{28}$ Talmud Bavlì, Hagigah 12a.

${ }^{29}$ Talmud Bavlì, Nedarim 39b. Voir aussi le traité Pessahim 54a, et Midrach Rabba. Genèse, Bernard MARUANI et Albert COHEN-ARAZI (trad.), Verdier, Lagrasse, «Les Dix paroles », 2010, chap. 1, p. 31-49.

${ }^{30}$ Moïse MAÏMOnide, Le Guide des égarés, éd. cit., II, 28, p. 208.
} 
conséquent, l'univers ne peut pas ne pas être, et la terre et les cieux sont donc éternels :

Il soutient [...] qu'aucune chose matérielle ne peut être produite sans une matière (préexistante), mais il soutient en plus que le ciel n'est aucunement sujet à la naissance et à la corruption. [...] il est, selon lui, de la catégorie de l'impossible que Dieu change son vouloir ou qu'il lui survienne une volonté nouvelle, et que tout cet univers, tel qu'il est, Dieu l'ait fait exister par sa volonté, sans pourtant qu'il ait rien fait du néant. De même [...] il s'ensuit par conséquent que tout cet univers, tel qu'il est maintenant, tel il a été de toute éternité et tel il sera à tout jamais. ${ }^{31}$

L'absolue négation de la liberté de la volonté divine se fonde sur l'impossibilité de tout changement dans l'essence de Dieu. Mais si Maïmonide se sert de plusieurs arguments aristotéliciens pour réfuter la première opinion, il ne peut pas ici accepter la négation. Les théories aristotéliciennes sur la physique et la cosmologie formeraient - selon notre auteur - un système cohérent, tout en présentant quelques «erreurs », comme dans le cas de la position de certaines planètes par rapport au soleil, l'existence des épicycles ou le nombre des sphères célestes ${ }^{32}$. Ces erreurs n'invalident pas le système aristotélicien, mais montrent plutôt les limites de la rationalité humaine qui ne peut, au-delà de la sphère de la terre, que formuler des conjectures :

Tout ce qu'Aristote a dit sur tout ce qui existe au-dessous de la sphère de la lune, jusqu'au centre de la terre, est indubitablement vrai [...]. Mais, à partir de la sphère de la lune et au-dessus, tout ce qu'Aristote dit ressemble, à peu de chose près, à des simples conjectures. ${ }^{33}$

Cette « conjecture » justifie de la manière la plus simple le monde tel qu'il est, mais ne peut être considérée que comme probable, étant privée de tout

\footnotetext{
${ }^{31}$ Ibid., II, 13, p. 112.

${ }^{32}$ Pour le soleil, voir ibid., II, 9 ; pour les épicycles, voir ibid., II, 24 ; pour les sphères célestes, voir ibid., II, 11.

${ }^{33}$ Ibid., II, 22, p. 179.
} 
fondement logique ${ }^{34}$. La réponse donnée à cette troisième opinion n'est donc pas aussi claire que les précédentes :

En effet, nous ne voulons pas maintenant établir que le monde a été créé ; mais ce que nous voulons, c'est (de montrer) qu'il est possible qu'il ait été créé. ${ }^{35}$

La distinction entre éternité (qadamut) et perpétuité (nichut) permet d'exclure l'éternité - du moment où elle impliquerait la présence d'un substrat coéternel à Dieu, à savoir le temps - tandis que le concept de perpétuité serait accepté, n'étant pas forcement incompatible avec la fonction du Dieu créateur. L'action divine doit en effet toujours dépendre de la volonté de Dieu, qui est totalement autoréférentielle, sans que l'homme puisse connaître la raison pour laquelle le monde a été créé tel qu'il est :

Quand la sagesse (divine) décide qu'une chose doit apparaître, elle a apparu; or, la sagesse de Dieu étant éternelle comme son essence, ce qui en résulte est (également) éternel [...]. De même que nous ignorons pourquoi sa sagesse a exigé que les sphères fussent (au nombre de) neuf, ni plus ni moins, $[. .$.$] de même nous$ ignorons pourquoi la sagesse $[\ldots]$ a fait que l'univers existât après ne pas avoir existé. ${ }^{36}$

À plusieurs endroits, Maïmonide - sur la base de l'analogie avec le Trône - rappelle que la création temporelle du monde n'implique pas sa future corruption, et ici la contradiction apparaît encore plus évidente : il considère l'univers au même niveau que le Trône, sans paraître noter que celui-ci, selon les sources rabbiniques, a été créé avant l'univers, avec les sept éléments. Cela permet de s'interroger légitimement sur la possibilité de concevoir la création comme éternelle. Puisque le Guide s'exprime dans

\footnotetext{
${ }^{34}$ Ibid., II, 15, p. 126.

${ }^{35}$ Ibid., II, 17, p. 137.

${ }^{36}$ Ibid., II, 18, p. 142-143. La comparaison de l'action divine avec une source infinie (ibid., II, 12, p. 101), tout en introduisant l'élément de la perpétuité, liée à l'idée de la source qui n'arrête jamais de couler, permet d'affirmer une fois encore que l'action divine ne répond à aucun type de nécessité.
} 
un langage qui se veut incompréhensible pour le peuple, on peut penser qu'il contient deux versions de la création, pour deux destinataires différents : l'opinion exotérique, selon laquelle le temps a été créé au moment de la création; et la version ésotérique, réservée aux seuls lecteurs avisés, qui conçoit la dérivation éternelle de l'univers à partir de Dieu.

Il est possible alors, pour régler la question, de trouver certains repères dans la correspondance entre les trois opinions sur la création et les trois opinions sur la prophétie ${ }^{37}$. Selon la première, Dieu peut faire de quiconque un prophète, sans que les vertus humaines n'entrent en considération. Cette opinion est partagée par les païens et les gens simples, alors que la deuxième, admise par les philosophes, conçoit la prophétie comme une perfection naturelle de l'homme, sans que Dieu ait le pouvoir d'intervenir. Selon la troisième opinion, la prophétie est une perfection naturelle de l'homme, mais dépend de la volonté divine. Maïmonide affirme que cette opinion est admise par « nous », sans clarifier davantage cette expression.

\section{Cosmogonie et prophétie}

Maïmonide lui-même nous laisse la possibilité de voir une correspondance entre les opinions sur la création et celles sur la $\operatorname{prophétie}^{38}$. Différents interprètes s'y sont attachés, proposant de nombreuses correspondances ${ }^{39}$; nous analyserons ici la correspondance

\footnotetext{
${ }^{37}$ Pour les opinions sur la prophétie, voir ibid., II, 32.

38 «Il en est des opinions des hommes sur la prophétie comme de leurs opinions concernant l'éternité ou la nouveauté du monde ; je veux dire que, de même que ceux pour qui l'existence de Dieu est avérée professent trois opinions (diverses) sur l'éternité ou la nouveauté du monde, comme nous l'avons exposé, de même aussi les opinions concernant la prophétie sont au nombre de trois » (ibid., II, 32, p. 259). Voir sur cette question Roberto GATTI, «Il filosofo e il profeta. Rassegna tematica di critica maimonidea », Eidos, 2 (2001), p. 59-84.

39 Lawrence KAPLAN ( Maimonides on the Miraculous Element in Prophecy », Harvard Theological Review, LXX, 3-4 (1977), p. 233-256) propose la correspondance
} 
proposée par Warren Z. Harvey ${ }^{40}$. Selon lui, la première opinion sur la création, à savoir la création à partir du non-existant, correspond à la première opinion sur la prophétie, selon laquelle Dieu peut faire de quiconque un prophète. L'analogie se fonde sur le fait que, dans les deux cas, Dieu agit par miracle. Toutefois, Maïmonide dit explicitement que cette opinion est suivie par les païens et les gens simples. Cela signifie que «tous ceux qui admettent la loi de Moïse ${ }^{41}-$ ceux qui admettent la création à partir du non-existant - seraient aussi ces gens simples. De cette manière, la création à partir du non-existant représenterait l'enseignement exotérique de Maïmonide, et non sa véritable opinion.

L'opinion selon laquelle Dieu crée à partir d'une matière éternelle correspondrait à la deuxième opinion sur la prophétie, qui conçoit cette dernière comme une perfection naturelle de l'homme. Les deux sont, en effet, admises par des philosophes non aristotéliciens et, dans les deux cas, Maïmonide présente la troisième opinion comme très proche de la seconde, mais avec une différence. Si ces opinions se ressemblent, en effet, c'est que dans les deux cas la prophétie est une perfection naturelle de l'homme; cependant, c'est de la volonté divine qu'elle dépend d'après la troisième opinion. De la même manière, cette troisième opinion sur la création est présentée comme similaire à la deuxième, avec une distinction : dans les deux cas on considère que rien ne peut venir du non-existant mais, selon la théorie aristotélicienne, les cieux ne sont pas sujets à la génération et à la corruption.

1:3, 2:1, 3:2, tandis que Herbert DAVIDSON («Maimonides' Secret Position on Creation », in Studies in Medieval Jewish History and Literature, Isadore TWERSKY (dir.), Cambridge (Mass.), Harvard University Press, « Harvard Judaic Monographs », 2, 1979, p. 16-40) propose la correspondance 1:1, 2:3, 3:2.

${ }^{40}$ Warren Zev HARVEY, «A Third Approach to Maimonides Cosmogony-Prophetology Puzzle », art. cit.

${ }^{41}$ Moïse MAÏMONIDE, Le Guide des égarés, éd. cit., II, 13, p. 104. 
Suivant ce raisonnement, les troisièmes opinions - l'éternité du monde et la prophétie dépendant de la volonté divine-doivent correspondre. Ce procédé permet de formuler l'hypothèse selon laquelle Maïmonide a pu soutenir la théorie aristotélicienne de l'éternité du monde, du moment qu'il écrit que cette troisième opinion est admise par «nous ».

Cependant, bien qu'il affirme que «tout ce qu'on prétend être une preuve de la nouveauté du monde est sujet aux doutes ${ }^{42}$, Maïmonide ne dit pas explicitement que la création éternelle a été démontrée. Il considère seulement celle-ci comme étant plus proche de la nature de ce qui existe :

La manière véritable, c'est-à-dire la méthode démonstrative dans laquelle il n'y a point de doute, consiste à établir l'existence de Dieu, son unité et son incorporalité par les procédés des philosophes, lesquels procédés sont basés sur l'éternité du monde. Ce n'est pas que je croie l'éternité du monde, ou que je leur fasse une concession à cet égard ; mais c'est que, par cette méthode, la démonstration devient sûre et on obtient une parfaite certitude sur ces trois choses, savoir, que Dieu existe, qu'il est un et qu'il est incorporel, sans qu'il importe de rien décider à l'égard du monde, (savoir) s'il est éternel ou créé. ${ }^{43}$

Le raisonnement paraît quelque peu embrouillé : notre auteur affirme ne pas croire à l'éternité du monde, pourtant celle-ci représente le seul moyen de démontrer l'unité de Dieu et de renier toute représentation anthropomorphique de la divinité. L'argumentation aristotélicienne qui démontre l'existence d'un premier moteur est à la base de la preuve maïmonidéenne de la nécessité de l'unité de Dieu, en tant que cause première. De plus, la réfutation de la corporéité de Dieu est un thème central du premier livre du Guide; la bataille contre les anthropomorphismes représente une préoccupation centrale de Maïmonide, les anthropomorphismes étant justement l'expression de la religiosité populaire. De ce fait, il est raisonnable de croire que l'éternité du monde

${ }^{42}$ Ibid., I, 71, p. 347.

${ }^{43}$ Ibid., I, 71, p. 348. 
représente son enseignement ésotérique, accessible uniquement à l'élite intellectuelle, tandis qu'une lecture superficielle pourrait se contenter de voir dans l'expression «tous ceux qui croient dans la loi de Moïse » ${ }^{44}$ l'acceptation du créationnisme classique.

En conclusion, face aux deux solutions radicalement opposées que proposent la philosophie et la religion et entre lesquelles il semble hésiter, Maïmonide opère une distinction précise. On revient à la question de la dichotomie soulevée par le doute, et qui est ici résolue puisque l'opposition ne s'avère qu'apparente, les deux visions appartenant à deux plans complètement indépendants : la loi s'adresse en fait au peuple et aux non-initiés, alors que les vérités philosophiques ne sont que l'apanage d'une élite érudite. De plus, déchiffrer le puzzle du Guide signifie repenser la problématique de la création du monde par des méthodes d'analyse qui impliquent des questions ne concernant pas la seule cosmogonie. À travers l'association entre la théorie de la prophétologie et le problème cosmogonique, il est possible de trouver une solution à la question de l'éternité du monde qui rende raison de la complexité du texte maïmonidéen. Une lecture linéaire, en effet, présenterait le risque d'une mauvaise compréhension, tandis que la méthode proposée par Maïmonide même, à savoir repérer des connexions entre chapitres différents, permet de mettre à jour la problématique d'une pensée qui ne correspond pas toujours à l'opinion de l'orthodoxie juive.

${ }^{44}$ Ibid., II, 13, p. 104. 